\title{
The Muonium Antimatter Gravity Experiment
}

\author{
Thomas J. Phillips ${ }^{1, *}$ (For the MAGE and muCool Collaborations) \\ ${ }^{1}$ Illinois Institute of Technology, Chicago, IL
}

\begin{abstract}
A direct measurement of the gravitational acceleration of antimatter has the potential to show that we live in a "Dirac-Milne" Universe, which could explain cosmological observations without the need for dark matter, dark energy, inflation, or missing antimatter. Such a measurement would also be sensitive to the possible existence of a fifth force. Cooling antimatter to temperatures where gravitational energies are comparable to thermal energies is challenging for most forms of antimatter, which annihilate upon contact with matter. The exception is the antimuon $\left(\mu^{+}\right)$, which is easily cooled by stopping in cold matter, but the short muon lifetime poses challenges. Positive muons that stop in material will combine with free electrons to form muonium, a neutral leptonic atom with most of its mass derived from the $2^{\text {nd }}$-generation antimuon. We are developing the Muonium Antimatter Gravity Experiment (MAGE) to measure the gravitational force on muonium using a novel, monoenergetic, low-velocity, horizontal muonium beam directed at an ultra-precise atom interferometer. If successful, MAGE will measure for the first time the gravitational coupling to a $2^{\text {nd }}$-generation particle in a system whose antimatter-dominated mass is not predominantly strong-interaction binding energy. The novel MAGE beam production approach could also have important applications to other muonium experiments as well as to the measurement of $g-2$.
\end{abstract}

\section{Introduction}

Muonium $(\mathrm{Mu})$ is an exotic hydrogen-like atom composed of a positive muon and an electron with unique properties that can be exploited to make numerous fundamental measurements. Since neither the muon nor the electron has any known internal structure, calculations of the bound state are free of the difficulties arising from the finite size of the nucleus in normal atoms. Precision spectroscopic measurements of muonium allow one to determine fundamental constants such as the muon mass, its magnetic moment and magnetic anomaly, and the fine structure constant [1]. The absence of sidereal and diurnal variations in muonium spectroscopic measurements provides stringent limits on CPT violation [1]. Muonium-antimuonium conversion is analogous to $K^{0}-\overline{K^{0}}, B^{0}-\overline{B^{0}}$, and neutrino oscillations and appears naturally in many beyond-the-Standard-Model theories [2]. In addition, cold muonium can be ionized to provide a source of cold muons, a method that is currently planned for the muon $g-2$ experiment under development at J-PARC [3, 4].

The Muonium Antimatter Gravity Experiment (MAGE) Collaboration aims to extend the list of fundamental measurements using muonium by measuring the gravitational force of the

\footnotetext{
*e-mail: phillips@PHYS.IIT.EDU
} 
earth on muonium [5]. Because the muonium mass is dominated by that of the antimuon, measuring the gravitational force on $\mathrm{Mu}$ will provide a direct measurement of the coupling of gravity to antimatter and to a second-generation particle, making the measurement uniquely sensitive to a possible fifth force that couples to the second generation [6].

Muonium experiments are limited by currently available Mu sources and would benefit from the development of better Mu sources in progress at Switzerland's Paul Scherrer Institute (PSI). Indeed, the gravity experiment most likely requires the development of a new $\mathrm{Mu}$ source as well as an ultra-precise interferometer. This paper describes these developments as well as the motivation for the gravity measurement.

\section{Motivation}

At NSF's and DOE's request, the U.S. High Energy Physics Advisory Panel produced a short list of the most important questions in particle physics [7]. It is intriguing that of those nine questions, the measurement of antimatter gravity may have relevance to more than half, and the potential to answer three. While perhaps a priori unlikely, an antimatter gravity experiment could show that our Universe is described by "Dirac-Milne" [8, 9] or lattice [10] cosmology, containing equal parts matter and antimatter that repel each other gravitationally. This would explain the mystery of the missing antimatter without the need for additional $C P$ violation. With a net gravitational mass of zero, the Universe would be flat and expanding linearly, which fits the Type Ia supernova data with no need of Dark Energy [9-11]. The slower initial expansion allows all parts of the visible Universe to be in thermal contact, resolving the horizon problem with no need for inflation $[9,10]$ and the age problem with no need for a cosmological constant [9]. Having both positive and negative gravitational mass results in gravitational vacuum polarization $[12,13]$, which provides a mechanism for Modified Newtonian Dynamics (MOND) [14, 15], and fits galactic rotation curves with no need of Dark Matter. Of course, the Equivalence Principle requires that the acceleration of antimatter on the earth $\bar{g}=g$, and indirect arguments based upon the virtual antimatter component in matter suggest that $\bar{g}=g$ with a high degree of accuracy [16]. However, we already have evidence that virtual particles do not interact gravitationally, for if they did the cosmological constant would be 120 orders of magnitude larger than observed [17]. If antimatter is identified with the $r<0$ solutions to General Relativity's Kerr-Newman equation (Kerr-Newman singularities have been suggested as a model for electrons [18-22]), then General Relativity would predict $\bar{g}=-g$ and virtual pairs of particles would feel no gravity, so the indirect limits on $\bar{g}$ would not apply. The potential for a single measurement to explain multiple mysteries, with no need to introduce the new physics of cosmic inflation, Dark Energy, and Dark Matter, provides ample motivation for the gravity measurement.

Several collaborations at CERN are conducting experiments that will attempt to measure the gravitational force on antimatter using antihydrogen [23-26], of which one has published broad limits on $\bar{g}$ [26]. However, these experiments are extremely challenging, in some cases requiring decades of work to get to the point where they can make and trap antihydrogen. A primary reason for this difficulty is that antiprotons and antihydrogen annihilate upon contact with ordinary matter. This renders making the cold antihydrogen needed for a gravity measurement particularly challenging. The experiments are made more challenging by the difficulty of getting enough antiproton beam time, a problem about to become worse as antiprotons will be unavailable during CERN's 2-year-long shutdown in 2019-2020. In addition, antihydrogen experiments suffer from the theoretical complication that the antiproton mass is dominated by binding energy so some have argued that a $1 \%$ measurement would not be sensitive to the mass of its antiquark constituents [27]. 


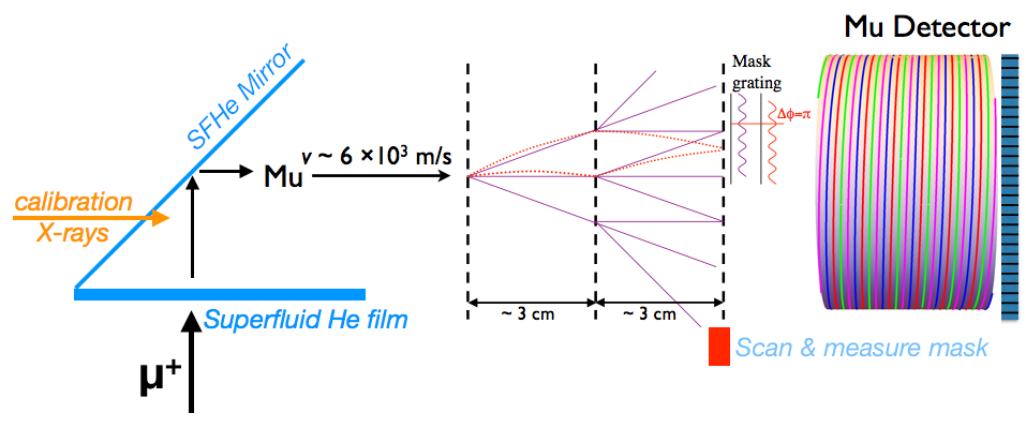

Figure 1. Principle of the MAGE experiment, shown schematically in elevation view (not to scale). Phase shift of $\pi$ shown for illustrative purposes only; actual phase shift will be small.

Antimuons $\left(\mu^{+}\right)$, on the other hand, do not annihilate on contact with ordinary matter, so they are easy to cool by stopping them in a cold material. Once stopped, they can combine with a free electron to form cold muonium. Making a gravity measurement with muonium will be enormously challenging, primarily because of the short $(2.2 \mu \mathrm{s})$ lifetime of the muon, but the challenges are quite different from the antihydrogen challenges, thus it is possible that the muonium measurement could turn out to be less challenging than the antihydrogen measurement. The Mu mass is $99.5 \%$ antimatter thus avoiding antihydrogen's theoretical complications. Additionally, the muonium experiment will be uniquely sensitive to gravitational and fifth-force couplings to second-generation particles [28-30].

\section{MAGE: The Muonium Antimatter Gravity Experiment}

The principle of MAGE, an application of well-established atom interferometry [31], is illustrated in Fig. 1. A horizontal muonium beam is directed into a three-grating interferometer. The first two gratings set up an interference pattern that has the same period as the gratings. Its phase is determined by scanning the third grating vertically by several grating periods and measuring the resulting sinusoidal variation in detected $\mathrm{Mu}$ intensity. Gravity causes a phase shift in the interference pattern which is equivalent to the deflection of an individual muonium atom. The interferometer is aligned using a soft X-ray beam with wavelength comparable to that of the Mu. This X-ray beam will also be used to determine the phase of an undeflected beam. The gratings are able to rotate with respect to the vertical for systematic studies; there is no gravitational phase shift when the grating slits are vertical.

\subsection{Muonium Beam}

Muonium is produced in many materials when a positive muon is stopped and a weakly bound electron is available to be captured. Since within the material, muonium behaves like a hydrogen atom, its transport is governed mainly by diffusion processes. Therefore in order to reach a high yield in vacuum the stopping target needs to be thin and/or porous, or the muons must be moved to the surface before they form muonium. Vacuum yields from 3 to $40 \%$ per stopped muon have been reached with various methods: tungsten foil heated to $2500 \mathrm{~K}$ [32], $\mathrm{SiO}_{2}$ powder [2, 33], laser-ablated $\mathrm{SiO}_{2}$ aerogel [34] or mesoporous $\mathrm{SiO}_{2}$ films [35]. For these existing muonium sources, $\mathrm{Mu}$ are emitted with a thermal velocity distribution and uniformly in $\cos \theta$ ( $\theta$ being the angle relative to the surface). This is inefficient for many 
applications, including the gravity measurement, which accepts Mu only within a small solid angle.

Fortunately, there is an alternative method for making muonium that will produce a beam ideal for the gravity experiment. The basis of this beam is that $\mathrm{Mu}$, which is a light isotope of hydrogen, has a negative chemical affinity in superfluid helium so when it diffuses out of the liquid, it, like hydrogen and deuterium [36], will be ejected with a velocity normal to the surface that is large compared to its thermal velocity.

The concept of forming a Mu beam using a film of superfluid helium (SFHe) was published in 2011 by Taqqu [37]. The formation of Mu in SFHe is a function of charge mobility, which grows exponentially below the $\Lambda$ point. Muonium formation efficiency in SFHe is near $100 \%$ at low temperature [38]. The chemical potential of hydrogen in superfluid helium depends upon the isotopic mass. These potentials have been calculated for hydrogen $(37 \mathrm{~K})$, deuterium (14 K), and tritium (7 K) [39], and experimentally verified for deuterium [36]. The corresponding potential for $\mathrm{Mu}$ is $270 \mathrm{~K}$ [37], so $\mathrm{Mu}$ will be ejected from the surface with a kinetic energy of $23 \mathrm{meV}$, which corresponds to a velocity of $6.3 \mathrm{~mm} / \mu \mathrm{s}$, much larger than its thermal velocity in the superfluid helium. For our application, the superfluid helium will be at $0.2 \mathrm{~K}$, so the energy dispersion will be $\approx 0.2 / 270=0.07 \%$, and the 1 -dimensional beam divergence $\approx \sqrt{0.2 / 270}=27 \mathrm{mrad}$.

Muonium that forms in the superfluid helium must reach the surface in order to be ejected into the vacuum. There are two methods that can ensure a large fraction of the Mu reaches the surface: either stop the muons in a very thin film so roughly half the Mu that forms will diffuse to the top surface, or stop the muons in a thick film and use an electric field to drift the muons to the top surface, seeded with electrons, where muons form $\mathrm{Mu}$ and are ejected. The MAGE Collaboration plans to investigate both of these methods.

\subsubsection{Ultra-Bright Muon Beam}

In order to stop muons efficiently in a thin film, they must be extremely low in energy and have a very small velocity dispersion. Conventional surface muon beams are much too hot for this, so the muCool project at PSI is developing a method for compressing the muon phase space by 10 orders of magnitude with an efficiency of $\approx 10^{-3}$, which will allow the muons to be stopped in a $1 \mu \mathrm{m}$ film of liquid helium. The project is based on ideas published by Taqqu in 2006 [40]: a $\mu^{+}$beam is stopped in helium gas where the muons are first compressed transverse to the original beam direction and then in the longitudinal direction before being extracted back into vacuum. A sketch of the setup is shown in Fig. 2. The compression is based on the position-dependent drift-velocity vector of a charged particle under the influence of electric (E) and magnetic (B) fields in the presence of a gas density gradient. The drift velocity $\mathbf{v}_{D}$ is given by

$$
\mathbf{v}_{D}=\frac{\mu E}{1+\omega^{2} \tau_{c}^{2}}\left[\hat{\mathbf{E}}+\omega \tau_{c} \hat{\mathbf{E}} \times \hat{\mathbf{B}}+\omega^{2} \tau_{c}^{2}(\hat{\mathbf{E}} \cdot \hat{\mathbf{B}}) \hat{\mathbf{B}}\right] .
$$

The effect of the helium gas at 5 mbar is characterized by the mobility of the muons and the mean time between collisions $\tau_{c} ; \omega=e B / m_{\mu}$ is the cyclotron frequency. In the experimental setup shown in Fig. 2 the electric and magnetic fields and the temperature distribution are arranged such that in the transverse compression stage (left side of figure) the muons drift to the right along the bottom wall (colder, higher density, first term of Eq. (1) dominating) and top wall (warmer, lower density, second term of Eq. (1) dominating) of the triangular cell, while in the longitudinal compression stage (center of figure) they follow the magnetic field and are compressed longitudinally (third term of Eq. (1) dominating). Finally the muons with 


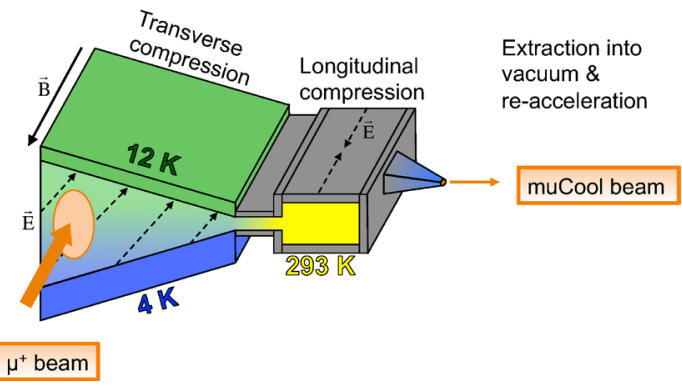

Figure 2. Schematic of the muCool apparatus. The surface muon beam is stopped in cold helium gas with a density gradient designed so that the $\mathbf{E} \times \mathbf{B}$ drift angle causes transverse compression. Electric field components in the $\mathbf{z}$ direction cause longitudinal compression. Cooled muons are extracted on the right.

an energy of about $1 \mathrm{eV}$ can be extracted into vacuum through a $1 \mathrm{~mm}$-diameter orifice. Once in the vacuum the muons can be re-accelerated. Extraction of the beam from the magnetic field must be done with care in order to avoid significantly increasing the phase space; an example of how this was done for positrons is described in [41].

The concept of longitudinal compression was demonstrated in an experiment conducted a few years ago [42]. More recently the muCool collaboration has demonstrated longitudinal compression within about $3 \mu$ s with an efficiency of approximately 100\% (apart from decay losses) $[43,44]$. The transverse compression stage is the more complicated as it requires a cryogenic helium gas cell. Transverse compression was demonstrated in 2015 [44].

Having successfully demonstrated both longitudinal and transverse compression, a new target which combines both types of compression at once is currently being tested. If successful, this will improve the efficiency of muCool by reducing the time required for cooling. The next steps will be to extract the cooled muons back into the vacuum, reaccelerate, and extract from the magnetic field.

\section{Ultra-Bright Muonium Beam Production}

The muCool $\mu^{+}$beam will be directed through a $30 \mathrm{~nm}$-thick silicon-nitride window and stopped inside a $1 \mu \mathrm{m}$-thick superfluid helium film at $0.2 \mathrm{~K}$. Roughly half of the formed $\mathrm{Mu}$ atoms reach the top surface of the film and are ejected with a velocity of $6.3 \mathrm{~mm} / \mu \mathrm{s}$. Due to the thinness of the helium film the original beam size is preserved and the emission into vacuum takes place with high efficiency. The resulting muonium beam is thus of sub-millimeter beam size, monochromatic $(\Delta E / E \approx 0.07 \%)$ and with a small divergence $(27 \mathrm{mrad})$. This vertical beam can be reflected to the horizontal by a SFHe-coated mirror [45], making it ideal for the gravity measurements, as the interferometer will be able to accept the entire beam. This beam will also be an ideal source for improving other fundamental measurements using muonium.

\section{Novel E-Field Muonium Source Using a Thick Superfluid Helium Film}

While the ultra-bright Mu beam being developed by the muCool program is nearly ideal for the gravity experiment, there is another possibility for developing an even more intense beam suitable for the gravity experiment, and this beam could be available without the technical risk associated with muCool. This is the method described by Taqqu in [37]. It differs from the thin-SFHe-film Mu source just described in that the $\mu^{+}$are stopped in a thick SFHe film and a surface layer of electrons draws them to the surface before making $\mathrm{Mu}$, substantially raising the efficiency for the Mu to be ejected into the vacuum. This method can utilize an uncompressed surface muon beam by using a thicker window to degrade the muons, and while the beam spot is larger than the nominal area of the gravity experiment, the mismatch for PSI's $\pi E 5$ beam is only a factor of 4 . This mismatch can be reduced by adjusting the dimensions 
of the interferometer and by focusing the muonium beam with a curved mirror [45] since the angular divergence of the beam is smaller than the interferometer's acceptance (which is roughly the requirement that the beam pass sequentially through two $1 \mathrm{~cm}$ squares separated by $\sim 6 \mathrm{~cm}$ ). The larger number of muons in the uncompressed surface muon beam more than compensates for the lower $\mu^{+}$stopping efficiency and interferometer acceptance (see Table 1 and Fig. 5 below), although redirecting the full surface muon beam upward will require more sophisticated beam optics than directing the muCool beam upward, a non-trivial difference.

This thick-film method of making a $\mathrm{Mu}$ beam requires preparing a layer of electrons on the upper surface of the superfluid helium. This well-established technique has been demonstrated in [46] with the electrons produced using a tungsten tip in the liquid helium; an external electric field drew them to the surface where they were trapped in a potential well [47]. An electric field of $100 \mathrm{~V} / \mathrm{cm}$ corresponds to a surface density just above $10^{8} \mathrm{e} / \mathrm{cm}^{2}$, a typical density for these experiments. The electrons are observed to remain at the surface for long times (minutes at low temperatures) after the voltage used to attract the electrons to the surface is removed [47, 48]. An electric field of $100 \mathrm{~V} / \mathrm{cm}$, created entirely by the surface electrons, will cause $80 \%$ of the stopping muons to drift away from the electrons left by their ionization trail [49]. In liquid helium, positive ions attract a "snowball" of helium atoms which determines their mobility $[48,50]$. The drift velocity at $100 \mathrm{~V} / \mathrm{cm}$ saturates near $42 \mathrm{~m} / \mathrm{s}$ [51], and at this velocity the muons that stop in the top $100 \mu \mathrm{m}$ will drift to the surface in 1 muon lifetime, combine with an electron, and be emitted as Mu. A simulation of a simple stainless degrader indicates it is possible to get $5 \%$ of a $29.8 \mathrm{MeV} / \mathrm{c}$ surface muon beam to stop in SFHe and drift to the surface before decaying, a number that can likely be improved with optimization. Using a surface muon beam such as PSI's $\pi E 5$ beam with $1.2 \times 10^{8} \mu / \mathrm{s}$, we could produce a beam with up to $4 \times 10^{6} \mathrm{Mu} / \mathrm{s}$, depending upon the duty cycle required to maintain the surface electrons. For the high-rate beams, electrons may be lost because of recombination with $\mathrm{He}^{+}$ions from muon ionization trails as well as $\mathrm{Mu}$ formation. In principle it should be possible to continuously resupply the surface electrons and eliminate a duty-cycle factor, but there are too many unknowns about SFHe physics to be confident about this before the development work is done.

\subsection{Muonium Interferometer}

The MAGE Collaboration plans to use an ultra-precise interferometer to measure the very small deflection the muonium beam experiences in a few muon lifetimes. This interferometer, currently under development at IIT [5] (see Fig. 3), will use a support structure machined from a single silicon block. This is a common technique used in X-ray optics and is particularly effective at cryogenic temperatures where silicon is extremely stable with an extremely small coefficient of thermal expansion.

The interferometer gratings will be mounted in frames that will be moved with piezoelectric actuators for alignment and scanning. The positions of the gratings will be monitored with semiconductor-laser tracking frequency gauges (SL-TFGs), developed by MAGE collaborators J.D. Phillips and R.D. Reasenberg [52]. SL-TFGs have sub-picometer accuracy, already achieved at IIT, more than sufficient for the interferometer.

The baseline MAGE design uses a $100 \mathrm{~nm}$ grating period since gratings with this period have been fabricated with adequate fidelity [53]. Gratings with a smaller period would improve the sensitivity of the experiment, so MAGE will use investigate fabricating gratings with finer periods and will use the smallest period that proves practical. With these gratings the interferometer will operate in the quantum regime. The optimum open fraction for the gratings will depend upon several design parameters, but is expected to be near $60 \%$ for the first grating and $40 \%$ for the second grating. These two gratings set up an interference pattern 

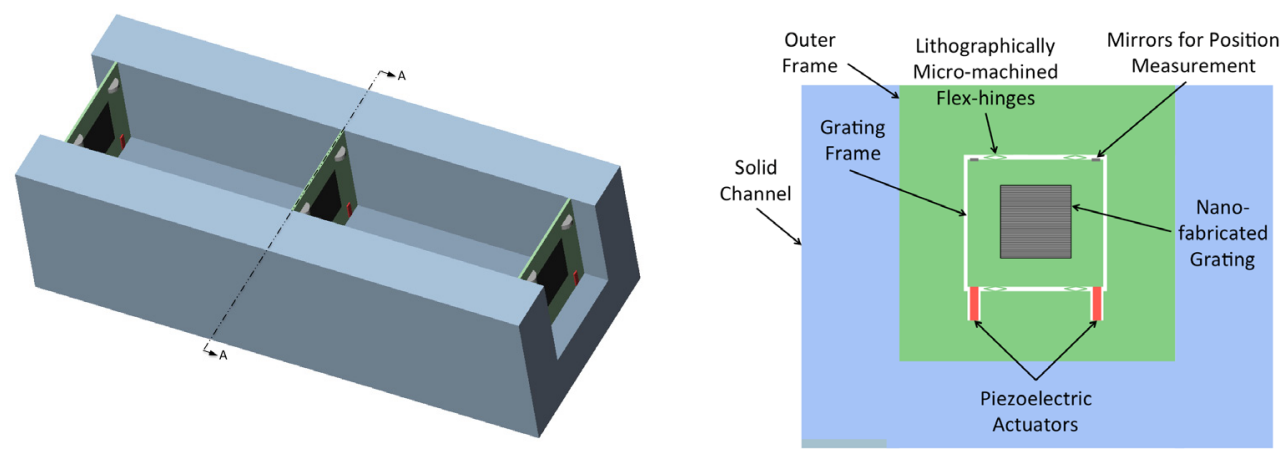

Figure 3. (left) CAD drawing of the muonium interferometer; (right) Section A-A. In blue is the support structure for the gratings, a solid channel machined out of a single silicon block. Each grating is mounted in a silicon frame connected to an outer frame by flex-hinges; a pair of piezoelectric actuators permits small rotations to align the gratings precisely in parallel, as well as scanning of the third grating. Each grating frame has mirrors (or possibly corner-cube retroreflectors) on its top corners that form part of the optical TFGs used to measure their position.

with the same period as the gratings. Since this pattern is too fine to image directly, the phase of the pattern is determined by measuring the transmission of muonium through a third grating of identical period as its position is scanned. An open fraction of $37 \%$ is optimum for the third grating [31], and while the maximum theoretical contrast is $20 \%$ or more, imperfections in the interferometer will reduce the contrast so a contrast of only $10 \%$ is assumed for the sensitivity calculations presented in this paper. The gravitational phase shift depends upon the Mu transit time, so a time-of-flight measurement would be useful for a thermal beam but unnecessary for the mono-energetic Mu beam produced by SFHe.

\subsection{Muonium Detector}

When Mu decays, the decaying muon produces an energetic positron and a low-energy electron is left behind. The signal for the MAGE Mu detector will be the coincidence of signals from scintillators measuring the positron and a hit in an electron detector. This will allow discrimination of $\mathrm{Mu}$ decays occurring before the third interferometer grating from those occurring after the third grating, since the electric field accelerating the electron will be nonzero only after the third grating. Only $1.5 \%$ of the muonium entering the interferometer will live long enough to make it past the third grating, so detecting the electron will be important for background rejection for tracks that could have originated before or at the third grating.

A baseline design for the muonium detector's positron tracker is shown in Fig. 4. Hits in the tracker's fibers and paddles localize the Mu decay when combined with the electron location in the transverse plane. The electron from the Mu decay will be accelerated by an electric field onto a position-sensitive detector on the far end. G4beamline simulations [54] indicate that this baseline design would accept $66 \%$ of muonium decays from the signal region with only $0.1 \%$ background from the much more numerous decays in the interferometer. Simple changes, such as extending the outer tracker $2 \mathrm{~cm}$ over the interferometer and adjusting the incident muon's polarization, increase the acceptance to $72 \%$. 


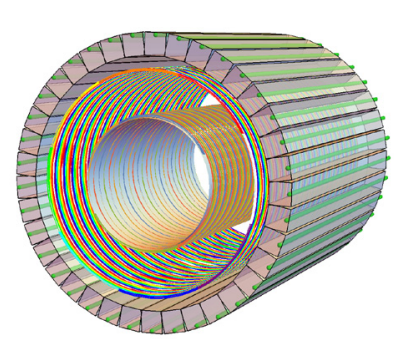

Figure 4. Muonium detector comprised of two cylinders, each having scintillating fibers wound helically around a support tube. The length of each fiber will be approximately one attenuation length, so by reading out both ends of each fiber it will be possible using timing and/or pulse-height differences to determine where the fiber was hit with enough precision to determine the coordinate along the tube with a resolution of a fiber diameter. Azimuth $(\phi)$ will be measured using scintillating paddles with embedded wave-shifting fibers (read out at one end). Electrodes in the center provide an electric field to accelerate the electron onto a detector (not shown).

\section{The MAGE Measurement}

The challenge of MAGE is to make a significant gravity measurement within the time permitted by the muon's $2.2 \mu$ s lifetime. The phase shift $\phi$ of the interferometer is given by ([31] Eqn. 52):

$$
\phi=[\mathbf{G} \cdot \mathbf{a}+2 \mathbf{G} \cdot(\mathbf{\Omega} \times \mathbf{v})] t^{2}
$$

where $\mathbf{G}$ is the grating vector (in the plane of the grating, perpendicular to its lines, with $|\mathbf{G}|=2 \pi / d$ ) for a grating with period $d$, a is the acceleration of the Mu (e.g. due to gravity), $\boldsymbol{\Omega}$ is the rotation of the interferometer (e.g. due to the earth's rotation), $\mathbf{v}$ is the velocity of the $\mathrm{Mu}$, and $t$ is the transit time of the Mu between gratings. The sensitivity $S$ of the interferometer to the gravitational acceleration for $N_{0}$ detected $\mathrm{Mu}$ is [55]:

$$
S=\frac{1}{C \sqrt{N_{0}}} \frac{d}{2 \pi} \frac{1}{t^{2}}
$$

where $C$ is the interferometer's contrast. The sensitivity of the gravity measurement is optimized by using finest grating pitch (smallest $d$ ) consistent with high fabrication fidelity. The contrast has a theoretical maximum of $C=29.5 \%$ for a three-grating interferometer without separated beams [56], but grating imperfections, misalignments, vibrations, beam coherence characteristics, the lack of collimation, etc., will reduce this contrast so for the calculation (below) we assume $C=10 \%$. We assume $1 \mathrm{~cm}(5 \mathrm{~cm})$ separation between the Mu source and the interferometer for the thermal (SFHe) beam, and we assume $d=100 \mathrm{~nm}$; gratings with this period have been fabricated but not yet with the $1 \mathrm{~cm}^{2}$ area assumed here.

We use a full calculation (as opposed to a Monte Carlo simulation) of the Mu interferometer in order to optimize the interferometer's sensitivity for the gravity measurement. For a given $\mathrm{Mu}$ velocity, a longer interferometer will increase $t$ but reduce $N_{0}$ due to the muon's finite lifetime. Figure 5 shows, as a function of the interferometer's separation between gratings, how much beam is required to acquire sufficient statistics to reduce the statistical uncertainty for the gravitational acceleration measurement to $4 \mathrm{~m} / \mathrm{s}^{2}(=0.4 \mathrm{~g}$, to get a $5 \sigma$ sign measurement assuming $|\bar{g}|=g$ ) using PSI's $\pi E 5$ beam for three different Mu sources: an existing aerogel thermal source, a thin-film SFHe source using the muCool beam, and the thick-film SFHe source. Note that this is actual beam time not including dead time for triggers or any operations that cannot be done while the beam is running, which may include calibrating the interferometer with X-rays, preparing and maintaining the SFHe, etc. Table 1 shows results using the optimum grating separation.

In order to study the systematic uncertainties of the interferometer, it will be important to measure a known signal. It may be possible to do this using the Sagnac effect, measuring the phase shift due to the earth's rotation. This effect results from the interferometer not being in an inertial reference frame and moving slightly during the Mu transit time. The size of the effect is dependent upon the direction of the beam (e.g. relative to north) and the orientation 

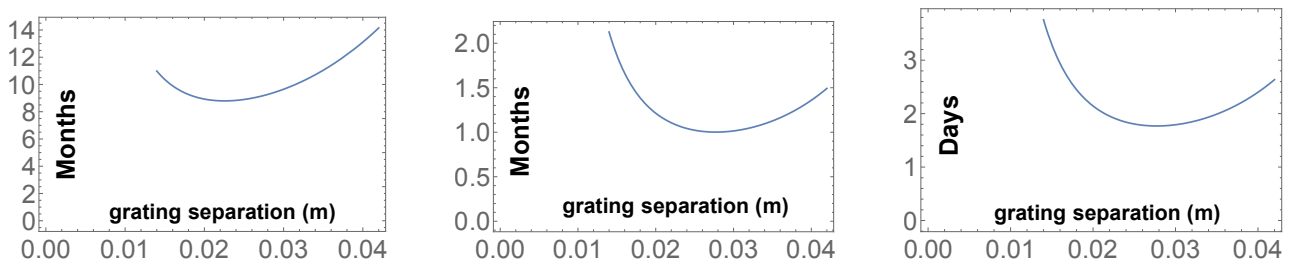

Figure 5. The plot on the left shows beam time required to acquire sufficient statistics to make a measurement with a statistical uncertainty of $0.4 \mathrm{~g}$ using PSI's $\pi E 5$ beam for a thermal beam vs. the separation between interferometer gratings. The center plot is the same when using the ultra-bright thin-film superfluid $\mathrm{He}$ (muCool) beam. If the novel thick-film Mu source test is successful, the beam time required for a sign determination could be reduced further, as shown on the right plot. (These plots do not include corrections for systematic uncertainties, which will likely be worse for lower Mu rates.) Here the distance from the Mu production target is assumed to be $1 \mathrm{~cm}$ for the thermal beam but $5 \mathrm{~cm}$ for the SFHe target, since for the SFHe target the Mu beam must be reflected from vertical to horizontal.

Table 1. Estimated performance of Mu sources for the gravity measurement using PSI's $\pi E 5$ beam line. Acceptance is for an interferometer with $1 \mathrm{~cm}^{2}$ gratings, and Beam Time is that required to acquire sufficient statistics for a $\bar{g}$ measurement with a statistical uncertainty of $4 \mathrm{~m} / \mathrm{s}^{2}$, sufficient to

determine the sign of $\bar{g}$ with $5 \sigma$ uncertainty if its magnitude is $g$. (These times do not include corrections for systematic uncertainties, which will likely be worse for lower Mu rates.) These results indicate that a rate of about $10^{4} \mathrm{Mu} / \mathrm{s}$ or more in the interferometer's acceptance would enable MAGE, so the SFHe Mu sources need not reach their full potential for this project to succeed.

\begin{tabular}{|c|c|c|c|c|}
\hline Mu Source & $\mu$ rate & Mu rate & MAGE Acceptance & Beam Time $(5 \sigma)$ \\
\hline \hline Thermal $(300 \mathrm{~K})$ & $1.2 \times 10^{8} / s$ & $1 \times 10^{6} / s$ & $5 \times 10^{-4}$ & 6400 hours \\
\hline muCool SFHe & $1.2 \times 10^{5} / s$ & $5 \times 10^{4} / s$ & 1 & 720 hours \\
\hline thick-film SFHe & $1.2 \times 10^{8} / s$ & $4 \times 10^{6} / s$ & 0.25 & 43 hours \\
\hline
\end{tabular}

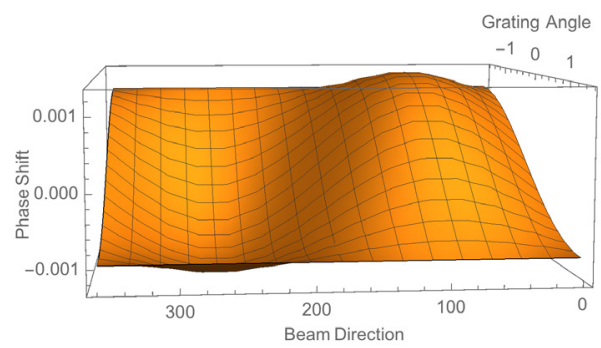

Figure 6. Sagnac effect: Phase shift vs. the (compass) direction of the muonium beam and the angle of the grating relative to horizontal.

of the gratings relative to the rotation axis, as can be seen from the second term in Eqn. 2 . This second term is plotted as a function of these two variables in Fig. 6. This effect is small, only about $10 \%$ the size of the gravitational phase shift, as can be seen in Fig. 7. However, it may be measurable if systematic uncertainties can be sufficiently controlled and the thick-film SFHe source works at or near its full potential. The statistical uncertainty on a single data point is shown in Fig. 7 for 18 days of beam time using the thick-film SFHe source assuming its full potential. This effect could also be studied using a conventional atomic beam in order to understand systematic effects of the interferometer. 


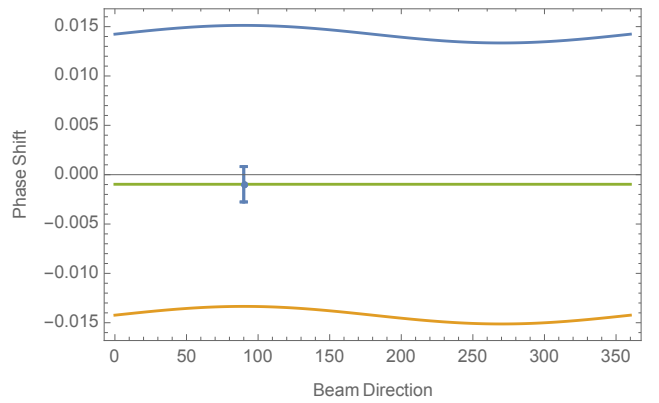

Figure 7. Phase shift vs. the compass direction of the muonium beam including both gravity and the Sagnac effect due to the earth's rotation. The top and bottom curves are for $\bar{g}= \pm g$ with horizontal interferometer gratings, while the center curve is for vertical interferometer gratings where there is no gravitational phase shift. The data point indicates the statistical uncertainty that would be achieved with 18 days of beam time assuming the full potential of the thick-film SFHe muonium source.

\section{Conclusion}

It is troubling that the sole raison d'être for what comprises $95 \%$ of the Universe in standard cosmology is to make the $5 \%$ we can observe fit the predictions of General Relativity. Despite considerable effort to find it, there is little evidence for Dark Matter, Dark Energy, or inflation beyond the cosmological observations for which their existence is postulated. Antimatter gravity has the potential to explain the biggest mysteries in physics and cosmology without the need to introduce this new physics and without missing antimatter. Efforts to measure antimatter gravity using antihydrogen are underway, but are challenging because antiprotons and antihydrogen annihilate upon contact with matter, making them difficult to cool. An alternate approach to measuring antimatter gravity uses muonium, which can be made cold but decays quickly due to the muon's lifetime. The MAGE collaboration is developing novel muonium beams and an ultra-precise interferometer to enable the measurement of the gravitational force on muonium. This is an extremely challenging experiment, but it has the potential to fundamentally change our understanding of the Universe.

\section{References}

[1] K.P. Jungmann, Journal of the Physical Society of Japan 85, 091004 (2016), https://doi.org/10.7566/JPSJ .85.091004

[2] L. Willmann, P.V. Schmidt, H.P. Wirtz, R. Abela, V. Baranov, J. Bagaturia, W. Bertl, R. Engfer, A. Großmann, V.W. Hughes et al., Phys. Rev. Lett. 82, 49 (1999)

[3] Tech. rep., J-PARC (2011), https://g2sakura.kek.jp/public/doc/ MCDR-submit.pdf

[4] S. Kanda, Nuclear Physics B - Proceedings Supplements 253, 212 (2014), the Twelfth International Workshop on Tau-Lepton Physics (TAU2012)

[5] D.M. Kaplan, E. Fischbach, K. Kirch, D.C. Mancini, J.D. Phillips, T.J. Phillips, R.D. Reasenberg, T.J. Roberts, J. Terry, ArXiv 1601, 07222 (2016)

[6] T.D. Lee, C.N. Yang, Phys. Rev. 98, 1501 (1955)

[7] P. Drell, A. Albrecht, S. Aronson, K. Baker, J. Bagger, N. Calder, E. Gates, F. Gilman, J. Jackson, S. Kahn et al., Tech. rep., DOE/NSF (2006), http://www . quantumuniversereport.org/

[8] A. Benoit-Lévy, G. Chardin, Astronomy \& Astrophysics 537, A78 (2012)

[9] A. Benoit-Lévy, G. Chardin, International Journal of Modern Physics: Conference Series 30, 1460272 (2014)

[10] M. Villata, Astrophysics and Space Science 345, 1 (2013)

[11] J.T. Nielsen, A. Guffanti, S. Sarkar, Scientific Reports 6, 35596 EP (2016) 
[12] L. Blanchet, Classical and Quantum Gravity 24, 3529 (2007)

[13] D.S. Hajdukovic, Astrophys Space Sci 334, 215 (2011)

[14] J. Bekenstein, M. Milgrom, Astrophys. J. 286, 7 (1984)

[15] S.S. McGaugh, Phys. Rev. Lett. 106, 121303 (2011)

[16] D.S.M. Alves, M. Jankowiak, P. Saraswat, ArXiv 0907, 4110 (2009)

[17] S. Weinberg, Rev. Mod. Phys. 61, 1 (1989)

[18] G. Chardin, Hyp. Int. 109, 83 (1997)

[19] H.I. Arcos, J.G. Pereira, Gen. Rel. Grav. 36, 2441 (2004)

[20] A. Burinskii, Gravitation and Cosmology 14, 109 (2008)

[21] A. Burinskii, Journal of Physics: Conference Series 361, 012032 (2012)

[22] M. Villata, Annalen der Physik 527, 507 (2015)

[23] G.D. et al. (57 authors), Tech. Rep. CERN-SPSC-2007-017, CERN (2007), http:// cds.cern.ch/record/1037532

[24] G. Gabrielse, P. Larochelle, D.L. Sage, B. Levitt, W. Kolthammer, R. McConnell, P. Richerme, J. Wrubel, A. Speck, M. George et al., Phys. Rev. Lett. 100, 113001 (2008)

[25] G. Dufour, D.B. Cassidy, P. Crivelli, P. Debu, A. Lambrecht, V.V. Nesvizhevsky, S. Reynaud, A.Y. Voronin, T.E. Wall, Adv. High Energy Phys. 2015, 379642 (2015)

[26] A.E. Charman, C. Amole, M. Ashkezari, M. Baquero-Ruiz, W. Bertsche, E. Butler, A. Capra, C. Cesar, M. Charlton, S. Eriksson et al., Nature Communications 4, 1785 (2013)

[27] FNAL PAC, Physics Advisory Committee Meeting March 27-29, 2008 Comments and Recommendations, (and private communication), http: //www.fnal.gov/directorate/program_planning/Mar2008PACPublic/ Mar2008PACReportPublic.pdf

[28] A. Kostelecky, J. Tasson, ArXiv 1006, 4106 (2010)

[29] J.D. Tasson, Int. J. Mod. Phys. Conf. Ser. 30, 1460273 (2014)

[30] J.D. Tasson, Symmetry 8, 111 (2016)

[31] A.D. Cronin, J. Schmiedmayer, D.E. Pritchard, Rev. Mod. Phys. 81, 1051 (2009)

[32] A.P. Mills, J. Imazato, S. Saitoh, A. Uedono, Y. Kawashima, K. Nagamine, Physical Review Letters 56, 1463 (1986)

[33] G. Beer, G. Marshall, G. Mason, A. Olin, Z. Gelbart, K. Kendall, T. Bowen, P. Halverson, A. Pifer, C. Fry et al., Phys. Rev. Lett. 57, 671 (1986)

[34] G. Beer, Y. Fujiwara, S. Hirota, K. Ishida, M. Iwasaki, S. Kanda, H. Kawai, N. Kawamura, R. Kitamura, S. Lee et al., Progress of Theoretical and Experimental Physics 2014 (2014), http://ptep.oxfordjournals .org/content/2014/9/091C01 . full .pdf+html

[35] A. Antognini, P. Crivelli, T. Prokscha, K. Khaw, B. Barbiellini, L. Liszkay, K. Kirch, K. Kwuida, E. Morenzoni, F.M. Piegsa et al., Phys. Rev. Lett. 108, 143401 (2012)

[36] M.W. Reynolds, M.E. Hayden, W.N. Hardy, Journal of Low Temperature Physics 84, $87(1991)$

[37] D. Taqqu, Phys. Procedia 17, 216 (2011)

[38] R. Abela, D. Herlach, E. Morenzoni, G. Solt, U. Zimmermann, F. Gygax, A. Schenck, D. Eshchenko, E. Krasnoperov, E. Meillikhov, JETP Lett. 57, 157 (1993)

[39] M. Saarela, E. Krotscheck, Journal of Low Temperature Physics 90, 415 (1993)

[40] D. Taqqu, Phys. Rev. Lett. 97, 194801 (2006)

[41] D. Gerola, W.B. Waeber, M. Shi, S.J. Wang, Review of Scientific Instruments 66, 3819 (1995), http://dx.doi.org/10.1063/1.1145443 
[42] Y. Bao, A. Antognini, W. Bertl, M. Hildebrandt, K.S. Khaw, K. Kirch, A. Papa, C. Petitjean, F.M. Piegsa, S. Ritt et al., Phys. Rev. Lett. 112, 224801 (2014)

[43] A. Eggenberger, I. Belosevic, G. Wichmann, Development of a low-energy, highbrightness $\mu^{+}$beam line, in Proceedings of the Seventh Meeting on CPT and Lorentz Symmetry (CPT'16) (Indiana University, Bloomington, 2016), http://arxiv.org/ abs/1607.06206

[44] A. Eggenberger, Ph.D. thesis, ETH Zurich (2016)

[45] V.G. Luppov, W.A. Kaufman, K.M. Hill, R.S. Raymond, A.D. Krisch, Physical Review Letters 71, 2405 (1993)

[46] C. Barenghi, C. Mellor, C. Muirhead, W. Vinen, Journal of Physics C: Solid State Physics 19, 1135 (1986)

[47] W. Schoepe, G. Rayfield, Z. Naturforsch. 26a, 1392 (1971)

[48] J. Poitrenaud, F. Williams, Phys. Rev. Lett. 29, 1230 (1972)

[49] D.G. Eshchenko, V.G. Storchak, J.H. Brewer, G.D. Morris, S.P. Cottrell, S.F.J. Cox, Phys. Rev. B 66, 035105 (2002)

[50] K. Atkins, Phys. Rev. 116, 1339 (1959)

[51] M. Kuchnir, J. Ketterson, P. Roach, Phys. Rev. A 6, 341 (1972)

[52] R. Thapa, J.D. Phillips, E. Rocco, R.D. Reasenberg, Opt. Lett. 36, 3759 (2011)

[53] R.E. Grisenti, W. Schöllkopf, J.P. Toennies, J.R. Manson, T.A. Savas, H.I. Smith, Phys. Rev. A 61, 033608 (2000)

[54] T.J. Roberts, G4beamline: A "swiss army knife" for geant4, optimized for simulating beamlines., http://g4beamline.muonsinc.com/

[55] M. Oberthaler, Nuc. Inst. Meth. B 192, 129 (2002)

[56] T.D. Hammond, Ph.D. thesis, MIT (1997), http://hdl .handle.net/1721 .1/46044 